\title{
Importancia de los estándares intelectuales del pensamiento crítico en estudiantes de educación superior
}

\section{Importance of the intelectual standards of critical thinking in Higher Education students}

\author{
Edgar Cardoso $^{1}$, Mirian Duran $^{1, \risingdotseq}$, Patricia Ortega ${ }^{2}$ \\ 1 Universidad Andina del Cusco, Cusco, Perú. \\ 2 Universidad Nacional de San Antonio Abad del Cusco \\ ”mdurand@uandina.edu.pe
}

\begin{abstract}
Resumen
El pensamiento es un proceso mental que desarrolla toda persona a partir de razonamientos; es inherente al ser humano; pero pese a ello, muchos estudiantes que inician estudios superiores presentan dificultades en el desarrollo de diferentes procesos mentales, especialmente aquellos que están dirigidos a los estándares intelectuales del pensamiento crítico. Así, mencionamos algunas deficiencias en la falta de comprensión, análisis de argumentos, dificultades al no presentar una mentalidad abierta a diferentes opiniones; estándares esenciales en los estudiantes que dirigen organizaciones o trabajen con recursos humanos. Por lo que se hace necesario describir cuál es el nivel de estándares intelectuales que presentan los estudiantes ingresantes a las Escuelas Profesionales de Ciencias Administrativas, Contables y Económicas de la ciudad de Cusco. La presente investigación fue descriptiva no experimental. Para la recolección de datos se aplicó un cuestionario con 40 ítems para describir 6 estándares intelectuales a 120 estudiantes ingresantes. En el análisis de datos se aplicaron estadígrafos descriptivos. Los resultados permitieron identificar un nivel entre medio y bajo en los estándares intelectuales; por lo que es necesario aplicar estrategias que impulsen a los estudiantes a mejorar estos estándares del pensamiento crítico.
\end{abstract}

Palabras Clave: pensamiento crítico, estándares intelectuales, claridad, criterio lógico, profundidad, veracidad, exactitud

\begin{abstract}
Thought is a mental process that develops every person from reasoning; it is inherent in the human being; But despite this, many students who begin higher education have difficulties in developing different mental processes, especially those that are aimed at the intellectual standards of critical thinking. Thus, we mention some deficiencies in the lack of understanding, analysis of arguments, difficulties in not presenting an open mind to different opinions; essential standards in students who run organizations or work with human resources. For what is necessary to describe is the level of intellectual standards presented by incoming students to the Professional Schools of Administrative, Accounting and Economic Sciences of the city of Cusco. The present investigation was descriptive not experimental. For the data collection, a questionnaire with 40 items was applied to describe 6 intellectual standards to 120 incoming students. In the data analysis descriptive statistics were applied. The results allowed us to identify a level between medium and low in intellectual standards; Therefore, it is necessary to apply strategies that encourage students to improve these standards of critical thinking.
\end{abstract}

Keywords: critical thinking, intellectual standards, clarity, logical criteria, depth, truthfulness, accuracy

Citar como: Cardoso, E., Duran, M., Ortega, P.,(2019), Importance of the intelectual standards of critical thinking in Higher Education students. Rev Yachay, 8(1), 607-611.

Recibido: 14-09-2019; Aceptado 20-12-2019; Publicado 12-12-2019

\section{Introduccion}

Toda profesión busca desarrollar competencias que consideren habilidades relacionadas a cada especialidad, como el tener una comunicación eficaz, razonar críticamente, sustentar con argumentos precisos, ser consciente de la selección de mejores alternativas para la solución de problemas; habilidades necesarias en un mundo globalizado. La era del conocimiento, exige que los estudiantes 
desarrollen capacidades desde los primeros semestres de formación; esto debido a que muchos ingresantes llegan a la universidad con deficiencias; como, por ejemplo, las dificultades que presentan en identificar ideas esenciales en el discurso oral y escrito, sustentar con argumentos válidos, así como dificultades en trabajos cooperativos. Ya Facione (2007) indicaba que la misma historia nos demostró cuan peligroso es formar estudiantes que no saben buscar la verdad, que solo viven por vivir, sin realizar ningún tipo de cuestionamientos; solo son meros sobrevivientes bajo el influjo de opresores.

Un aspecto fundamental en la formación profesional es el desarrollo de los estándares intelectuales del pensamiento crítico, pues estos hacen que las personas sean razonables en diferentes circunstancias, y al hacer uso de estas de manera consciente, el pensamiento se vuelve claro, exacto, preciso, importante, amplio y justo; todo lo necesario para ser profesionales idóneos.

Los estándares intelectuales que corresponden al pensamiento crítico buscan el ser conscientes de qué estándares intelectuales son necesarios para evaluar diferentes discursos, tanto orales como escritos. Según Paul y Elder (2007), entre los estándares intelectuales necesarios para examinar diferentes situaciones se consideran: claridad, exactitud, precisión, relevancia, profundidad, amplitud, lógica, importancia y justicia.

1. Claridad y exactitud al evaluar diferentes discursos. Los mismos que ayudan a entender con facilidad el contenido; así como permiten que la información esté libre de distorsiones. Esta debe ir acompañada de ejemplos, y más datos sobre la información.

2. Emplear criterio lógico en diferentes situaciones. El contenido de la información deber estar libre de contradicciones, tener sentido en la enunciación, mantener la interrelación da párrafo con párrafo y estar acorde a las evidencias o argumentos; además de presentar la debida exactitud, es decir evidencias y que puedan ser verificables.

3. Identificar si un razonamiento es profundo y amplio. Necesariamente todo texto debe conllevar a interrogantes como ¿cuáles son las dificultades?, ¿conlleva complicaciones? Además, es necesario examinar el contenido desde diferentes perspectivas, tomando en cuenta otros puntos de vista.

4. Uso de estándares intelectuales para examinar las propias ideas. Cada uno debe ser consciente de qué tipo de mensaje presenta y si estos son coherentes con los estándares intelectuales. Lo que uno expresa, ¿tiene que ver con el problema? ¿ayuda en algo al asunto tratado? ¿tendré algún interés personal en el asunto? ¿respeto el punto de vista ajeno?

5. Ser consciente de la veracidad, falsedad o contradicción de un razonamiento. Para lo cual es necesario considerar si el contenido resalta lo esencial, se enfoca en lo más importante; y si se presentan varios datos ¿cuál de ellos es lo más esencial?

El desarrollo de los estándares intelectuales en los estudiantes es importante, porque como ya se vio, ayudan en el análisis de información que pueda presentarse, tanto en lo oral como en lo escrito; de esta manera, los estudiantes que trabajen con recursos humanos serán capaces, no solo de dirigir, sino también de realizar trabajo cooperativo.

\section{Material y métodos}

La investigación tuvo un diseño no experimental, no se manipularon las variables, solo se recurrió a describirlas. El universo estuvo constituido por 1430 estudiantes ingresantes de la Universidad Andina del Cusco. La población estuvo constituida de 436 estudiantes; y la muestra de 120 estudiantes correspondientes a las Escuelas Profesionales de Administración, Negocios Internacionales, Economía, Contabilidad, Marketing y Finanzas. El tamaño muestral corresponde al grupo de estudiantes que enseñaron los investigadores durante el semestre 2018-I.

Para el procesamiento y análisis de datos se utilizaron los programas IBM SPSS Statics 24.0 y Microsoft Excel. Se adecuó el instrumento de Águila (2014), basado en la propuesta de Paul y Elder (2007). La validez del cuestionario se dio a nivel interno por dos especialistas de la Universidad en la que se realizó la investigación.

El instrumento evalúa los estándares intelectuales del pensamiento crítico, los mismos que presentan 41 items para evaluación de 6 estándares.

\section{Resultados}

El cuestionario fue aplicado a estudiantes varones y mujeres ingresantes al primer semestre de estudios correspondientes a las escuelas de Administración, Negocios Internacionales, Economía, Contabilidad, Marketing y Finanzas, cuyas edades fluctúan entre 17 y 19 años. Los resultados que alcanzaron los estudiantes de las diferentes escuelas fueron:

En el ítem 1: identifica estándares intelectuales al evaluar textos orales o escritos se verifica que son los estudiantes de Administración de Negocios Internacionales quienes en un 53.8\% demuestran identificar los estándares intelectuales; siguen los estudiantes de Economía con un 40.0\%; luego Contabilidad, 30.7\%; Administración, 26.6\%; Finanzas, $20.0 \%$ y Marketing con un $0.0 \%$

En el ítem 2: Evalúa con claridad y exactitud textos orales o escritos, se observa que Administración de Negocios Internacionales sobresale con un 38.4\%; le sigue Contabilidad con un 23.0\%; Economía, 20.0\%; Administración, 6.6\%; mientras que, Finanzas y Marketing, $0.0 \%$ 
Tabla 2

Estándares Intelectuales

\begin{tabular}{|c|c|c|c|c|c|c|c|}
\hline $\mathbf{N}^{\circ}$ & Items & $\begin{array}{l}\text { Administración } \\
\text { de Negocios } \\
\text { Internacionales }\end{array}$ & Administración & Contabilidad & Economía & Finanzas & Marketing \\
\hline 1 & $\begin{array}{l}\text { Identifica estándares } \\
\text { intelectuales al evaluar } \\
\text { textos orales o escritos. }\end{array}$ & $53.8 \%$ & $26.6 \%$ & $30.7 \%$ & $40.0 \%$ & $20.0 \%$ & $0.0 \%$ \\
\hline 2 & $\begin{array}{l}\text { Evalúa con claridad y } \\
\text { exactitud textos orales } \\
\text { o escritos. }\end{array}$ & $38.4 \%$ & $6.6 \%$ & $23.0 \%$ & $20.0 \%$ & $0.0 \%$ & $0.0 \%$ \\
\hline 3 & $\begin{array}{l}\text { Emplea criterio lógico } \\
\text { en } \quad \text { diferentes } \\
\text { situaciones. }\end{array}$ & $7.6 \%$ & $6.6 \%$ & $46.1 \%$ & $20.0 \%$ & $0.0 \%$ & $16.6 \%$ \\
\hline 4 & $\begin{array}{ll}\text { Identifica si un } \\
\text { razonamiento } & \text { es } \\
\text { profundo y amplio. } & \end{array}$ & $38.5 \%$ & $0.0 \%$ & $7.6 \%$ & $40.0 \%$ & $20.0 \%$ & $16.6 \%$ \\
\hline 5 & $\begin{array}{lr}\text { Usa } & \text { estándares } \\
\text { intelectuales } & \text { para } \\
\text { examinar sus propias } \\
\text { ideas. }\end{array}$ & $23.0 \%$ & $20.0 \%$ & $53.8 \%$ & $40.0 \%$ & $20.0 \%$ & $0.0 \%$ \\
\hline 6 & $\begin{array}{l}\text { Es consciente de la } \\
\text { veracidad, falsedad o } \\
\text { contradicción de un } \\
\text { razonamiento. }\end{array}$ & $46.1 \%$ & $13.3 \%$ & $30.7 \%$ & $26.6 \%$ & $0.0 \%$ & $0.0 \%$ \\
\hline
\end{tabular}

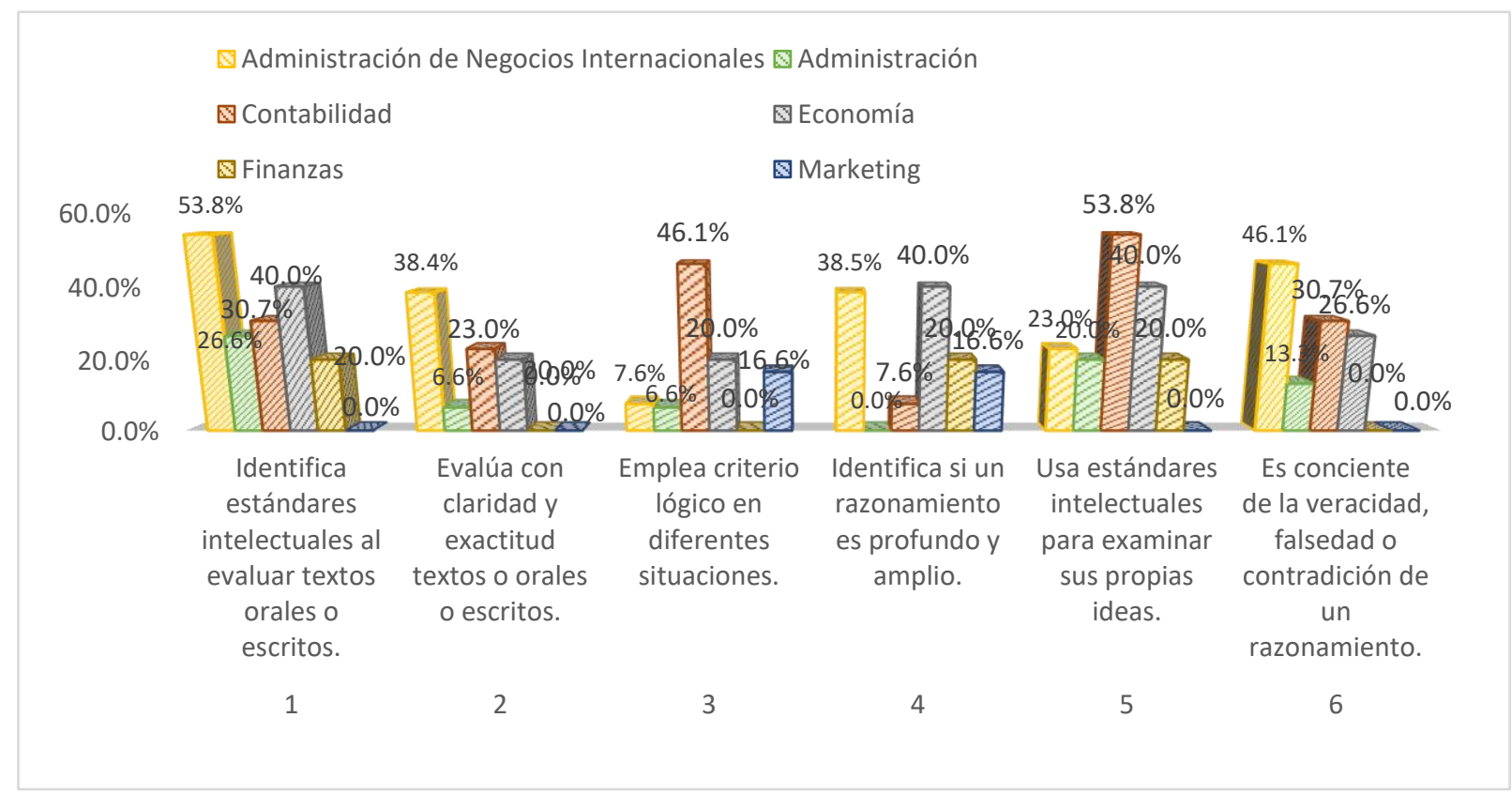

Figura 1. Estrategias intelectuales 
En el ítem 3: Emplea criterio lógico en diferentes situaciones, los estudiantes de Contabilidad alcanzaron un 46.1\%, Economía, 20.0\%; Marketing, 16.6\%; Administración de Negocios Internacionales, 7.6\%; Administración, 6.6\%; y Finanzas, 0.0\%

En el ítem 4: Identifica si un razonamiento es profundo y amplio, Economía alcanzó un 40.0\%; Administración de Negocios Internacionales, 38.5\%; Finanzas, 20.0\%; Marketing, 16.6\%; Contabilidad, 7.6\%; y Administración 0.0\%

En el ítem 5: Usa estándares intelectuales para examinar sus propias ideas, fueron los estudiantes de Contabilidad, quienes alcanzaron un 53.8\%; Economía, 40.0\%; Administración de Negocios Internacionales, 23.0\%; Administración y Finanzas, 20.0\%; y Marketing, $0.0 \%$

En el ítem 6: Ser consciente de la veracidad, falsedad o contradicción de un razonamiento: los estudiantes de Administración de Negocios Internacionales alcanzaron 46.1\%; Contabilidad, 30.7\%; Economía, 26.6\%; Administración, 13.3\%; Finanzas y Marketing, $0.0 \%$

\section{Discusión}

En la investigación “Aplicación de estándares intelectuales de evaluación por alumnos de la Universidad de Sonora” de Esperanza Águila (2012), se manifiesta la importancia de que el joven universitario ponga en juego todas las habilidades del pensamiento. Por lo que es importante estimular la enseñanza del modelo de pensamiento crítico de manera explícita. Tener un pensamiento crítico significa que alguien tiene la capacidad para juzgar una situación adecuadamente, no sólo en función de una mente estructurada y lógica, sino también con base en unos valores y principios éticos y en un manejo adecuado de las emociones, tal como menciona Espíndola (2005) en Moreno (2012).

Así, como indica la autora, es necesario aplicar estrategias que ayuden a desarrollar los estándares del pensamiento crítico, para ello se deben proponer modelos explícitos que coadyuven a dicha habilidades.

Parra (2013), en su tesis "Desarrollo de habilidades de pensamiento crítico en estudiantes" manifiesta la necesidad de priorizar estrategias que mejoren el análisis y argumentación, los cuales son primordiales en el pensamiento crítico.

Los resultados hacen ver que muchos alumnos no demuestran tener comprensión ni argumentan adecuadamente, por lo que, es imprescindible desarrollar actividades que mejoren estos estándares del pensamiento.

Es importante la aplicación de actividades tendientes al desarrollo de habilidades del pensamiento, porque son parte esencial en la formación de todo profesional, tal como se indica en la tesis de Cornejo, et al. (2017), se indica la idea de visibilizar la habilidad de pensamiento crítico de una manera más integrada. Es decir, considerar las habilidades cognitivas y metacognitivas como base y por otro lado la posibilidad de generar autorregulación y motivación a fin de lograr una disposición crítica, que implica estar abierto a enfoques múltiples para tomar decisiones e intervenir en la realidad social. Estos componentes definirían el pensamiento crítico como un proceso sofisticado y complejo, que incluiría habilidades cognitivas, de metacognición, disposición al juicio crítico, y colaboración frente a resolución de problemas.

\section{Conclusiones}

Primera. Los estudiantes presentan dificultades para identificar estándares intelectuales al evaluar textos orales o escritos.

Segunda. La claridad y exactitud en los textos orales o escritos es indispensable para lograr una buena comprensión por lo que las diferentes especialidades deben aplicar estrategias para lograr un nivel alto.

Tercera. No existe un $100 \%$ de estudiantes que logren demostrar criterio lógico, ni realizan razonamientos profundos y amplios o examinar sus propias ideas.

Cuarta. Es importante aplicar estrategias que conduzcan a superar el desarrollo de estándares intelectuales del pensamiento crítico.

\section{Referencias BIbliograficas}

Águila, E. (2012). Aplicación de estándares Hernández. L., (13 de Setiembre de 2008). ¿Por Molina, C., Morales, G.,Valenzuela, J. (2015). intelectuales de evaluación por alumnos. $R E D E X, 15$ qué enseñar a Pensar? Recuperado el 05 de diciembre del 2015.

Bernat de la Rosa, C. M. (2007). (2007) Miranda, C, Zambrano, F, Jelvez, M. (2010). Pensamiento Crítico: ¿Qué es y por qué ¿Incide la formación inicial en el es importante? Recuperado el 15 de junio desarrollo del pensamiento crítico de los de 2015, de estudiantes de pedagogía? Boletín de http://www.eduteka.org/PensamientoCrit icoFacione.php

Campos Arenas, A. (2007). Pensamiento Crítico: técnicas para su desarrollo (1era ed.). Bogota, Colombia: Magisterio. investigación educacional, 24, 79-89 http://www.scielo.edu.uy/scielo.php?script=sci nlinks\&pid=S168822120170001000190 $\underline{0027 \& \operatorname{lng}=\text { en }}$
Competencia transversal pensamiento crítico: Su caracterización en estudiantes de una secundaria de México. Revista Electrónica Educare (Educare Electronic Journal) EISSN: 1409-4258 Vol. 20, enero-abril, 2016: 1-26

Montoya, J. (2005). Estrategias didácticas para fomentar el pensamiento crítico en el aula. Tesis de Maestría. Universidad Católica del Norte. 
Ossa-Cornejo, Carlos J., Palma-Luengo, Maritza Paul, R. \& Elder, L. (2007). Pensamiento crítico. R., Lagos-San Martín, Nelly G., Quintana-Abello, Ingrid M., \& DíazLarenas, Claudio H. (2017). Análisis de Paul, R. \& Elder, L. (2003). Fundamentos del instrumentos de medición del pensamiento analítico. Sonora State pensamiento crítico. Ciencias University. En www.criticalthinking.org 19-28 Parra (2013) . Desarrollo de habilidades de https://dx.doi.org/10.22235/cp.v11i2.134 $\underline{3}$ de Maestría. Universidad de Guayaquil. de medición del pensamiento crítico. Scielo, 8 .

Roca, J. (2013). El desarrollo del pensamiento crítico a través de diferentes metodologías docentes en el Grado en Enfermería. Tesis de Maestría. Universidad de Barcelona.

pensamiento crítico en estudiantes. Tesis Sierra, J; Carpintero, E.; Pérez, L. (2010).

Palma, M., Lagos, N., Quintana, I., Díaz, C., \& Ossa, C. (2017). Análisis de instrumentos
Pensamiento crítico y capacidad intelectual. Vol. $15 \mathrm{n}^{\circ} 17,98-110$. Unirioja 